\title{
Crisis management during anaesthesia: pneumothorax
}

\author{
A K Bacon, A D Paix, J A Williamson, R K Webb, M J Chapman
}

Qual Saf Health Care 2005;14:e18 (http://www.qshc.com/cgi/content/full/14/3/e18). doi: 10.1136/qshc.2002.004424

See end of article for authors' affiliations .....................

Correspondence to: Professor W B Runciman, President, Australian Patient Safety Foundation, GPO Box 400, Adelaide, South Australia, 5001 Australia; research@apsf. net.au

Accepted 12 January 2005
Background: Pneumothorax is a potentially dangerous condition which may arise unexpectedly during anaesthesia. The diagnosis is one of exclusion, as initial changes in vital signs (cardiorespiratory decompensation and difficulty with ventilation) are non-specific, and other causes of such changes are more common, whereas local signs may be difficult to elicit, especially without full access to the chest.

Objectives: To examine the role of a previously described core algorithm "COVER ABCD-A SWIFT $\mathrm{CHECK}^{\prime \prime}$, supplemented by a specific sub-algorithm for pneumothorax, in the management of pneumothorax occurring in association with anaesthesia.

Methods: Reports of pneumothorax were extracted and studied from the first 4000 incidents reported to the Australian Incident Monitoring Study (AIMS). The potential performance of the structured approach, using the combination of algorithims described above for each of the relevant incidents, was compared with the actual management as reported by the anaesthetists involved.

Results: Pneumothorax was noted as a possible diagnosis in 65 reports; 24 cases had a confirmed pneumothorax, of which 17 were in association with general anaesthesia. It was considered that, correctly applied, the application of the algorithms would have led to earlier recognition of the problem and/or better management in $12 \%$ of cases.

Conclusion: Any pneumothorax may become a dangerous tension pneumothorax with the application of positive pressure ventilation. Limited access to the chest during anaesthesia may compromise the diagnosis. Recognition of any preoperative predisposition to a pneumothorax (for example, iatrogenic or traumatic penetrating procedures around the base of the neck) and close communication with the surgeon are important. Aspiration diagnosis in suspected cases and correct insertion of a chest drain are essential for the safe conduct of anaesthesia and surgery.
D iagnosis of pneumothorax during general anaesthesia is difficult, especially as this problem is uncommon. Although there are a number of well recognised high risk situations, pneumothorax may occur unexpectedly in a patient with no known risk factors. A small, undiagnosed, asymptomatic pneumothorax may become a tension pneumothorax on instituting positive pressure ventilation of the lungs after induction of or during anaesthesia. As the systemic signs of pneumothorax are non-specific and the local signs difficult to elicit if access to the chest is restricted, a high level of suspicion and a systematic approach is required for the rapid detection of this potentially dangerous problem.

In 1993, a "core" crisis management algorithm represented by the mnemonic COVER ABCD-A SWIFT CHECK (the AB precedes COVER for the non-intubated patient) was proposed as the basis for a systematic approach to any crisis during anaesthesia where it is not immediately obvious what should be done, or where actions taken have failed to remedy the situation. ${ }^{1}$ This was validated against the first 2000 incidents reported to the original Australian Incident Monitoring Study (AIMS). AIMS is an ongoing study which involves the voluntary, anonymous reporting of any unintended incident which reduced, or could have reduced, the safety margin for a patient. ${ }^{2}$

It was concluded that if this algorithm had been correctly applied, a functional diagnosis would have been reached within 40-60 seconds in 99\% of applicable incidents, and the learned sequence of actions recommended by the COVER portion would have led to appropriate steps being taken to handle the $60 \%$ of problems relevant to this portion of the algorithm. ${ }^{1}$ This study also showed that the $40 \%$ of problems represented by the remainder of the algorithm, ABCD-A SWIFT CHECK, were not always promptly diagnosed or appropriately managed. ${ }^{1-3}$ It was decided that it would be useful, for these problems, to develop a set of sub-algorithms in an easy to use crisis management manual. ${ }^{4}$ This study reports on the potential place of the COVER ABCD-A SWIFT CHECK algorithm in the diagnosis and initial management of pneumothorax, provides an outline of a specific crisis management algorithm for pneumothorax during anaesthesia, and provides an indication of the potential value of using this structured approach.

\section{METHODS}

Of the first 4000 incidents reported to AIMS, those which made reference to "pneumothorax" were extracted and analysed for relevance, presenting features, causes, diagnosis, management, and outcome. The COVER ABCD-A SWIFT CHECK algorithm, described elsewhere in this set of articles, ${ }^{4}$ was applied to each relevant report to determine the stages at which the problem might have been diagnosed and to confirm that activating the COVER portion would have led to appropriate initial steps being taken. As pneumothorax is not adequately dealt with by this algorithm, a specific subalgorithm for pneumothorax was developed (see figure), and its putative effectiveness was tested against the reports. How this was done is described elsewhere in this set of articles. ${ }^{4}$ The potential value of this structured approach (that is, the application of COVER ABCD-A SWIFT CHECK to the diagnosis and initial management of the problem, followed by the application of the pneumothorax sub-algorithm) was assessed in the light of AIMS reports by comparing its potential effectiveness for each incident with that of the actual management, as recorded in each report.

\section{RESULTS}

Of the 4000 reports sent to AIMS, 65 mentioned pneumothorax as a potential or actual diagnosis, and in 24 , a 


\section{PNEUMOTHORAX}

\section{SIGNS (1)*}

Difficulty with ventilation/respiratory distress

Desaturation

Hypotension

Heart rate changes

Unilateral chest expansion

Expose, inspect, palpate (2)

Auscultate, percuss

Abdominal distension

Distended neck veins, raised CVP

Tracheal deviation

\section{PRECIPITATING FACTORS (3)}

Any needle or instrumention, even days previously (4)

In or near the neck or chest wall

Down the trachea/bronchial tree

External cardiac massage

Fractured ribs, crush injury

Blunt trauma/deceleration injury

Problem with pleural drain already sited

Airway overpressure, obstructed ETT

Emphysema or bullous lung disease

\section{EMERGENCY MANAGEMENT}

Inform the surgeon

Inspect the abdomen, or the diaphragm from below if visible

Insert an IV cannula into the affected side (5)

Turn off the nitrous oxide

Insert a pleural drain at the same site (6)

Continuously observe the bottle for bubbling and/or swinging

Be vigilant for further deterioration in the patient, it may be due to: Increased or continuing air leak

Kinked/blocked/capped/clamped underwater seal drain

Contralateral pneumothorax

Misplaced pleural drain tip

Trauma caused by drain insertion

Misconnection of drain apparatus

The sub-algorithm forms a facing page of the

Crisis Management Manual 12.

* Numbers in brackets refer to Notes in the right hand panel.

\section{FURTHER CARE}

If the problem persists, consider cardiac tamponade.

Consider pericardiocentesis and/or opening the chest.

Arrange a chest $X$-ray and look for:

state of re-expansion of the lung

position of the tip of the drain. See (6) and (7) below.

Explain the nature of the problem to the patient before discharge from the recovery ward.

\section{NOTES:}

(1) The diagnosis is one of exclusion.

In $63 \%$ of 65 incidents where it was considered, it was not the cause. $71 \%$ of confirmed pneumothoraces occurred under general anaesthesia: detection in this sub-group was aided by - desaturation $41 \%$, hypotension $47 \%$.

Note: clinical observation is NOT reliable. See (2).

The commonest cause of unilaterally decreased breath sounds is endobronchial intubation.

(2) A sign for tension pneumothorax described in the Russian literature should be specifically sought. This involves detection by palpatation of widening of the intercostal spaces on the affected side.

Demonstration of the sign requires that the patient be positioned symmetrically. The tips of one or two fingers are then inserted in the mid axillary line and a comparison is made from one side to the other at an identical level. The wider side has the pneumothorax. Ref: Tsarev NI, Pugachev AF, Shelest Al. Diagnosis and treatment of spontaneous pneumothorax. Voen Med Zh 1987;8:51-2.

(3) Following chest wall/clavicular region blocks $-25 \%$. Whilst under general anaesthesia - 53\%. Of the GA sub-group: Post CVC insertion $-41 \%$. Associated with tracheostomy/base of neck procedures $24 \%$.

(4) Cronen MC, Cronen PW, Arino P, Ellis K. Delayed pneumothorax after subclavian vein catheterisation and positive pressure ventilation. $\mathrm{Br} J$ Anaesth 1991;67:480-2.

(5) Do not wait for confirmation by chest X-ray if the patient is rapidly deteriorating. Insert the cannula just cephalad to the third rib in the midclavicular or midaxillary line, and swiftly withdraw the needle as its tip may lacerate a moving lung.

(6) Emergency management of severe trauma (EMST) recommends underwater seal drain placement just anterior to the midaxillary line, as this avoids the internal mammary artery which may be punctured using the mid-clavicular approach, and allows drainage of blood.

Always use blunt dissection to penetrate the parietal pleura. Use of finger to sweep away any structures near the opening and then insert the drain.

(7) It should be noted that a chest X-ray may not detect a non-tension pneumothorax in a supine patient. Inspiratory AP and lateral views are preferable; a CT scan is the definitive test.

These notes comprise a reverse side of a page of the Crisis Management Manual ${ }^{12}$.

Figure 1 Pneumothorax.

pneumothorax was confirmed. The other 41 reports had no pneumothorax confirmed on chest $x$ ray examination. Of the positive diagnoses, 17 were associated with general anaesthesia, including two that were known prior to anaesthesia, and six were associated with concomitant nerve blocks in the chest wall or clavicular regions. Other cases included an intercostal drain mishap in a ward and a tracheostomy under local infiltration anaesthesia. The 17 confirmed cases during general anaesthesia (GA) were analysed for this study.

Almost half (seven of 17) of the cases of confirmed pneumothorax under GA occurred after insertion of central venous catheters, and a quarter (four of 17) in association with tracheostomy or other operations at the base of the neck. The remainder involved a supraclavicular brachial plexus block under GA, an intercostal block under GA, and an underwater seal intercostal drain that was not "bubbling".
The role of various monitors for detection of pneumothorax was also examined.

\section{Pulse oximetry}

Of the 17 GA reports, seven noted a drop in saturation. Of the 41 reports where pneumothorax was considered but there was no pneumothorax, the saturation dropped in 13. Thus pulse oximetry must be regarded as non-specific, ${ }^{5}$ as desaturation occurred in about $40 \%$ of cases in both groups.

\section{Blood pressure and pulse}

If a tension pneumothorax had occurred then a fall in blood pressure and a rise in pulse rate would be expected. The reports do not reliably identify tension as a specific item and thus both tension and non-tension pneumothoraces are incorporated as one group. In the 17 positive reports these blood pressure and pulse changes were documented in eight; 
in the negative reports they were noted five times-thus, they too must be regarded as non-specific signs.

\section{Central venous pressure}

Only one report mentioned a rise in central venous pressure (CVP). This could be expected in a tension pneumothorax due to the rise in intrathoracic pressure.

\section{Clinical observation}

The narrative in three of the positive reports commented on abdominal distension as a sign observed while the diagnosis was uncertain. This is worthy of being added to the clinical signs that raise a suspicion that there may be a pneumothorax.

When the COVER ABCD-A SWIFT CHECK algorithm was applied to each report, it was considered that the existence of a problem would have been detected at the Rl (review monitors) stage of COVER. However, it was considered that it would have been unlikely for the diagnosis of pneumothorax to have been made during the SCAN or CHECK phases of COVER. It was thought that a significant pneumothorax should be detected at the A stage of A SWIFT CHECK, (be Aware of Air and Allergy) in conjunction with the SWIFT CHECK portion of the algorithm.

When the potential effectiveness of the structured approach, represented by the COVER ABCD-A SWIFT CHECK algorithm and the special sub-algorithm for pneumothorax (see fig) was compared with that of the anaesthetist, as documented in each of the 17 incident reports, it was considered that properly applied, a structured approach would have led to a quicker and/or better resolution of the problem in two cases $(12 \%)$. In these cases, problems arose because of failure of communication between the surgeon and anaesthetist-principally, surgical perseverance with what turned out to be the wrong diagnosis.

\section{DISCUSSION}

Pneumothorax is an uncommon and potentially dangerous problem, especially during general anaesthesia, when the patient cannot complain of respiratory difficulty or pain, and with positive pressure ventilation, which increases the risk of a tension pneumothorax. ${ }^{6}$

The diagnosis of pneumothorax rests on thinking of the possibility whenever there are signs of circulatory or respiratory compromise or difficulty, especially in certain high risk situations (see box). The anaesthetist has an opportunity to diagnose a pneumothorax during inspection or examination of the chest during manual ventilation of the lungs (Vl, SCAN, or CHECK levels of COVER) or at $\mathbf{B}$ of $\mathrm{ABCD}$ in the spontaneously breathing patient. However, if the cardiorespiratory decompensation is modest, full exposure of the chest during an operative procedure may not be considered justifiable, and the diagnosis is less likely to be made.

Tradition has it that breath sounds should be diminished on auscultation, and that the trachea may be deviated away from the side of the pneumothorax. ${ }^{7}$ In practice, the commonest reason for an anaesthetised patient to have diminished breath sounds on one side is a mainstem bronchus intubation, ${ }^{5}$ and any displacement of the trachea is more likely to be due to a slight rotation of the head on the neck. Endobronchial intubation should routinely be excluded at the CHECK level, when the chest cannot be fully exposed, by the use of the test described by Pollard and Lobato. ${ }^{8}$

When the COVER ABCD algorithm has been completed at the CHECK level, and the cause of the problem is still not evident, the "four As" of A SWIFT CHECK must be specifically considered. In the "reminder" for the four As "Be Aware of Air and Allergy", "Air" serves as a reminder for
Box 1 Situations in which there is a risk of pneumothorax

- Procedures on or near the neck:

- central line insertion

- surgery

- brachial plexus block

- other blocks

- Procedures on or near the chest wall:

- intercostal block

- paravertebral block

- axillary block

- biopsies

- pericardial tap

- Instrumentation of the trachea/bronchi:

- tracheostomy

- tracheostomy tube change

- cricothyroid puncture

- difficult intubation

- bronchoscopy

- biopsy

- after deceleration injuries

- after external cardiac massage

- crush injuries

- Technical problems:

- overpressure of the airway

- obstructed endotracheal tube

- intercostal drain

misplaced

blocked

kinked

capped

clamped

valve misassembled

UWSD* tubes reversed

*UWSD, underwater seal drain.

Air in pleura (pneumothorax) as well as for Air (and other) embolism. Each of the four As each represents $0.5-1.5 \%$ of incident reports and they all share the feature that certain clinical signs and/or situations should immediately be searched for when certain combinations of changes are evident both on the monitors and clinically, and their cause has not become apparent during the COVER ABCD sequence.

The SWIFT CHECK portion of the algorithm may also play a role in the diagnosis of pneumothorax. The series reported here confirms findings in published case reports on pneumothoraces. Procedures after which there should be a high index of suspicion of pneumothorax include any instrumentation around the base of the neck (for example, insertion of a central venous catheter, a brachial plexus block, the creation of a tracheostomy, the manipulation or replacement of a tracheostomy tube) or in the region of the chest wall (for example, an intercostal block). To these may be added any instrumentation of the trachea or bronchial tree (for example, a biopsy) and any other blocks in the region of the neck and chest (for example, interscalene, paravertebral, 
and even attempted epidural blocks). Pneumothorax should always be considered after blunt trauma, in association with any significant deceleration injury, after external cardiac massage and with fractured ribs. It should be remembered that a small, previously undiagnosed, asymptomatic pneumothorax, which may have been present for many days may become much larger or become a tension pneumothorax with positive pressure ventilation of the lungs. Finally, when a pleural drain is in place, it should be checked that the tip is in the pleural space, that it is not kinked, blocked, capped, or clamped, and that any one way valve apparatus has been correctly assembled and orientated.

If pneumothorax is considered to be a possibility, the anaesthetist must go on to the ALERT-READY mode of COVER, which includes exposure of the chest and careful comparison of the left and right sides (see fig). A sign for tension pneumothorax described in the Russian literature should now be specifically sought. ${ }^{9}$ This involves detection by palpation of widening of the intercostal spaces on the affected side. Demonstration of the sign requires that the patient be positioned symmetrically. The tips of one or two fingers are then inserted in the mid-axillary line and a comparison is made from one side to the other at an identical level. The wider side has the pneumothorax. In concept, this is a logical follow on from the three records in this series that noted abdominal distension, and may be more reliable than the usual practice of simply inspecting the chest for unilateral distension.

If there is any suspicion that a pneumothorax may be responsible for the deterioration, then an urgent chest $x$ ray is indicated. If this is not available within a reasonable time, or the patient is deteriorating, either perform a needle aspiration of the pleural space, or insert a short intravenous cannula as a pleural space vent. For a needle aspiration, use a 10 or $20 \mathrm{ml}$ syringe containing approximately $3 \mathrm{ml}$ of water or saline and a $23 \mathrm{G}$ needle. Insert the needle just cephalad to the third rib in the mid-clavicular line, and aspirate. If there is a small stream of bubbles, this is a negative result: either the needle and syringe are loosely pushed together, or the tip of the needle is in lung tissue. If there are large bubbles ( $0.5 \mathrm{ml}$ or greater in estimated size) this is a positive result and there is a pneumothorax on that side. ${ }^{10}$ To place a pleural vent, insert a short intravenous cannula through the chest wall at the same site and withdraw the needle to avoid potential lung damage. If the anterior chest wall is unsuitable (for example, infection or extensive burns) insert the needle just cephalad to a rib high in the mid axillary line.

Following either of these tests, a pleural drain should be inserted at the same site, to minimise damage to the lung parenchyma or other underlying structures. If a pneumothorax is not suspected, but the patient's condition continues to deteriorate, the EMERGENCY mode of COVER should be applied, and the chest reassessed at regular intervals using the sequences outlined for the CHECK and ALERT-READY modes of COVER.

If the above tests are negative consider a diagnosis of pericardial tamponade. Cardiac tamponade should be considered with ongoing desaturation, tachycardia, hypotension, raised CVP, or distended jugular veins after pneumothorax has been excluded or drained. In these circumstances, if a pulse cannot be maintained with external cardiac massage, consideration should be given to opening the chest.

It is worth noting that a supine, antero-posterior chest $x$ ray may not detect a pneumothorax that has not proceeded to tension, but may be useful for diagnosis of a loculated pneumothorax in a patient with pleural adhesions. If the patient's condition permits, erect inspiratory and expiratory postero-anterior and lateral chest $x$ rays are advisable. If the clinical condition precludes this, head up, reverse Trendelenberg tilt, antero-posterior, and lateral chest $x$ rays may assist; computerised tomography, if available and logistically reasonable, will provide a definitive image. If there is chronic obstructive airways disease or pleural adhesions, or the patient is at risk of progressing to getting "stiff" lungs (for example, with multiple trauma or sepsis) insertion of a drain into lung parenchyma may have adverse consequences.

The current Early Management of Severe Trauma (EMST) protocol is that needle thoracocentesis should be performed in the mid clavicular line in the second intercostal space. ${ }^{11}$ Experience gained in prehospital care indicates that complications may ensue from passing a needle close to the inferior surface of the rib, where intercostal blood vessels may not be completely protected from trauma, or straying from the mid clavicular line close to the internal mammary artery. ${ }^{10}$ Hence, any needle or instrument should traverse the second intercostal space just above the cephalad border of the third rib, well away from the lateral sternal border. It is recommended by the EMST protocol that formal decompression through a drain tube is best performed just anterior to the mid axillary line; however, the site recommended for thoracocentesis is just as appropriate if access is easier (for example, during supine anaesthesia). A drain should be inserted by making a small incision to facilitate insertion of a blunt instrument-usually forceps-to penetrate the parietal

\section{Key messages}

- Twenty four confirmed cases of pneumothorax occurred amongst the first 4000 incidents reported to AIMS.

- Seventeen were associated with general anaesthesia (GA) and six with concomitant chest wall or clavicular region nerve blocks.

- Seven of the 17 associated with GAs occurred after insertion of central venous catheters and another four of the 17 with operations at the base of the neck (including tracheostomy).

- Pulse oximetry, blood pressure, and pulse changes must all be regarded as non-specific detectors of pneumothorax.

- High risk situations indicated from this series include: procedures on or near the neck or chest wall, instrumentation of the trachea/bronchi, chest trauma, and certain technical problems.

- Abdominal distension and also the reported Russian clinical observation of widening of the intercostal spaces on the affected side should both be added to the clinical signs that raise the suspicion of a pneumothorax.

- The diagnosis of pneumothorax rests upon thinking of the possibility whenever circulatory or respiratory compromise appears in the presence of high risk situations.

- A supine, antero-posterior chest $x$ ray may not detect a pneumothorax that has not proceeded to tension.

- Patient deterioration is an indication to perform either a needle aspiration or to place a pleural vent.

- It was considered that properly applied, a structured approach using the core algorithm and the specific pneumothorax sub-algorithm would have led to a quicker and/or better resolution of the problem in two of the $17 \mathrm{GA}$ cases. 
pleura, and then use a finger to sweep any structures away from the opening, before inserting the drain. Most drain tubes are supplied with a sharp stylet in the lumen of the drain tube which must be removed before use to avoid damage to underlying structures such as the lung, heart, diaphragm, spleen, liver, or major blood vessels.

In summary, in a patient with signs of circulatory or respiratory decompensation or difficulty, the cause of which remains obscure after going through the CHECK mode of the COVER ABCD-A SWIFT CHECK algorithm-especially in any of the risk situations listed in box 1 , the chest and abdomen should be fully exposed and a careful comparison made of both sides in accordance with the procedure outlined in the ALERT-READY mode of COVER. If a pneumothorax is suspected, and the patient is clinically stable, obtain an urgent chest $x$ ray. If a pneumothorax is confirmed insert a pleural drain. If the patient's condition warrants immediate treatment consider pleural aspiration or venting, as described above. This should be followed by an appropriately placed pleural drain at the same site. A loculated tension pneumothorax or cardiac tamponade should be considered if the needle test has been correctly performed and remains negative and the patient's condition continues to deteriorate.

Finally, before discharge from the recovery ward, the nature of the problem should be explained to the patient, the position of the tip of the drain and the state of the lung should be determined by chest $x$ ray, and arrangements for adequate analgesia and physiotherapy should be made. The problem should be clearly documented in the anaesthetic record. The patient should be reviewed by the anaesthetist, at appropriate intervals, until the lung is fully inflated and the drain has been safely removed.

\section{ACKNOWLEDGEMENTS}

The authors would like to thank all the anaesthetists in Australia and New Zealand who contributed to the 4000 incident reports upon which this and the other 24 papers in the Crisis Management Series are based. The coordinators of the project also thank Liz Brown for preparing the draft of the original Crisis Management Manual; Loretta Smyth for typing; Monika Bullock, RN, for earlier coding and classifying of data; Dr Charles Bradfield for the electronic version of the algorithms; Dr Klee Benveniste for literature research; Drs Klee Benveniste, Michal Kluger, John Williamson, and Andrew Paix for editing and checking manuscripts.
Authors' affiliations

A K Bacon, Consultant Anaesthetist, St John of God Hospital, Berwick, Victoria, Australia

A D Paix, Consultant Anaesthetist, Princess Royal University Hospital, Orpington, Kent, UK

J A Williamson, Consultant Specialist, Australian Patient Safety Foundation; Visiting Research Fellow, University of Adelaide and Royal Adelaide Hospital, Adelaide, South Australia, Australia

R K Webb, Senior Staff Specialist, Department of Anaesthesia and Intensive Care, The Townsville Hospital, Douglas, Queensland, Australia

M J Chapman, Senior Staff Specialist, Intensive Care Unit, Royal Adelaide Hospital and University of Adelaide, Adelaide, South Australia, Australia

This study was coordinated by The Australian Patient Safety Foundation, GPO Box 400, Adelaide, South Australia, 5001, Australia.

\section{REFERENCES}

1 Runciman WB, Webb RK, Klepper ID, et al. Crisis management: validation of an algorithm by analysis of 2000 incident reports. Anaesth Intensive Care 1993:21:579-92.

2 Webb RK, Currie M, Morgan CA, et al. The Australian Incident Monitoring Study: an analysis of 2000 incident reports. Anaesth Intensive Care 1993;21:520-8.

3 Webb RK, van der Walt JH, Runciman WB, et al. Which monitor? An analysis of 2000 incident reports. Anaesth Intensive Care 1993;21:529-42.

4 Runciman WB, Kluger MT, Morris RW, et al. Crisis management during anaesthesia: the development of an anaesthetic crisis management manual. Qual Saf Health Care 2005; 14:el

5 Runciman WB, Webb RK, Barker L, et al. The pulse oximeter: applications and limitations-an analysis of 2000 incident reports. Anaesth Intensive Care 1993;21:543-50.

6 Fossard JP, Samet A, Meistelman C, et al. Life-threatening pneumothorax of the ventilated lung during thoracoscopic pleurectomy. Can J Anaesth 2001;48:493-6.

7 In: Berkow R, Fletcher AJ, eds. In: Merck manual, 17th edn, Rahway, New Jersey: Merck and Company, 1999:541-3.

8 Pollard RJ, Lobato EB. Endotracheal tube location verified reliably by cuff palpation. Anesth Analg 1995;81:135-8.

9 Tsarev NI, Pugachev AF, Shelest Al. Diagnosis and treatment of spontaneous pneumothorax [In Russian]. Voen Med Zh 1987;8:51-2.

10 Bacon AK, Archer FL. A system for prehospital diagnosis of pneumothorax by ambulance officers. In: 6th World Conference on Emergency \& Disaster Medicine, edn. Hong Kong, September, 1989;(abstracts 2):15-16.

11 Trauma Committee, Royal Australasian College of Surgeons. Early management of severe trauma. Box Hill, Victoria: Capitol, 1992.

12 Australian Patient Safety Foundation. Crisis Management Manual: COVER $A B C D$ A SWIFT CHECK. Adelaide: Australian Patient Safety Foundation, 1996, 74. Available at http://www.apsf.net.au/anaesthesia.htm (accessed 6 September 2004). 